\title{
Some formulas for the restricted $r$-Lah numbers
}

\author{
Mark Shattuck \\ Institute for Computational Science \& Faculty of Mathematics and Statistics, \\ Ton Duc Thang University, Ho Chi Minh City, Vietnam \\ mark.shattuck@tdtu.edu.vn \\ Submitted May 12, 2018 - Accepted November 4, 2018
}

\begin{abstract}
The $r$-Lah numbers, which we denote here by $\ell^{(r)}(n, k)$, enumerate partitions of an $(n+r)$-element set into $k+r$ contents-ordered blocks in which the smallest $r$ elements belong to distinct blocks. In this paper, we consider a restricted version $\ell_{m}^{(r)}(n, k)$ of the $r$-Lah numbers in which block cardinalities are at most $m$. We establish several combinatorial identities for $\ell_{m}^{(r)}(n, k)$ and obtain as limiting cases for large $m$ analogous formulas for $\ell^{(r)}(n, k)$. Some of these formulas correspond to previously established results for $\ell^{(r)}(n, k)$, while others are apparently new also in the $r$-Lah case. Some generating function formulas are derived as a consequence and we conclude by considering a polynomial generalization of $\ell_{m}^{(r)}(n, k)$ which arises as a joint distribution for two statistics defined on restricted $r$-Lah distributions.

Keywords: restricted Lah numbers, polynomial generalization, $r$-Lah numbers, combinatorial identities
\end{abstract}

MSC: 11B73, 05A19, 05A18

\section{Introduction}

Sequences enumerating certain kinds of finite set partitions in which the smallest $r$ elements are required to belong to distinct blocks are often referred to as $r$-sequences. Examples that have been studied previously include the $r$-Stirling numbers $[4,14]$ of the first and second kind, $r$-Lah numbers [16] and $r$-derangement numbers [8, 20]. See also [15] for an $r$-generalization of the partial Bell polynomials. 
A restricted version of a counting sequence is one in which the block sizes of the underlying structure are at most a fixed number. Restricted Stirling numbers of both kinds (see, e.g., $[6,10,11]$ ) have been previously considered in conjunction with incomplete versions of the poly-Cauchy [9] and poly-Bernoulli [10] numbers. See [13] for a common polynomial analogue of the restricted Stirling and Lah numbers.

In this paper, we consider a generalization of the $r$-Lah numbers, denoted by $\ell_{m}^{(r)}(n, k)$, by requiring that no block size exceeds $m$. Note that $\ell_{m}^{(r)}(n, k)$ reduces to the classical Lah numbers (see, e.g., [12]) when $r=0$ and $m>n-k$. The limiting case as $m \rightarrow \infty$ coincides with the $r$-Lah numbers studied in $[1,3,16]$ and the configurations enumerated in this case are referred to as $r$-Lah distributions. See [17] for a related polynomial generalization and also [5] where $r$-Whitney-Lah numbers are introduced. Here, we will study $r$-Lah distributions with the added restriction that no block size exceeds $m$. This restriction causes the underlying counting sequence to behave somewhat differently than in the limiting case, which will be manifested by the formulas in the following sections. See [2] where $r$-Lah distributions are studied in which the block sizes are bounded from below.

This paper is organized as follows. In the next section, we make some preliminary definitions and find a basic recurrence satisfied by $\ell_{m}^{(r)}(n, k)$ where only the $n$ and $k$ parameters are changing. In the third section, we find some further recurrence formulas for $\ell_{m}^{(r)}(n, k)$ in which one or both of $m$ and $r$ are changing as well. Taking $m$ large in these formulas recovers prior $r$-Lah identities in some cases and apparently new identities for these numbers in others. We make use mostly of combinatorial arguments to establish our results, sometimes drawing upon the inclusion-exclusion principle and other times defining a direct bijection between the related structures. An explicit formula is derived in the fourth section in terms of binomial coefficients from which one obtains as a corollary an expression for the generating function. In the final section, a polynomial generalization of $\ell_{m}^{(r)}(n, k)$ is introduced and some of its properties discussed.

\section{Definition and basic recurrence}

If $m$ and $n$ are positive integers, then let $[m, n]=\{m, m+1, \ldots, n\}$ for $m \leq n$, with $[m, n]=\varnothing$ if $m>n$, the $m=1$ case of which will simply be denoted by $[n]$. Given $n, k, r \geq 0$ and $m \geq 1$, let $\mathcal{L}_{m}^{(r)}(n, k)$ denote the set of partitions of $[n+r]$ into $k+r$ contents-ordered blocks (i.e., lists) in which the elements of $[r]$ belong to distinct blocks and all blocks have size at most $m$. For example, if $n=m=2$ and $k=r=1$, then

$$
\mathcal{L}_{2}^{(1)}(2,1)=\{1 / 23,1 / 32,12 / 3,21 / 3,13 / 2,31 / 2\}
$$

We will refer to the elements of $[r]$ within a member of $\mathcal{L}_{m}^{(r)}(n, k)$ as special and use this also to describe the blocks to which they belong. Elements of $[r+1, r+n]$ and also the blocks composed solely of these elements will be referred to as non-special. 
For example, in $15 / 62 / 83 / 47 / 9 \in \mathcal{L}_{2}^{(2)}(7,3)$, the blocks 15 and 62 are special, while the blocks 83, 47 and 9 are non-special.

Let $\left|\mathcal{L}_{m}^{(r)}(n, k)\right|=\ell_{m}^{(r)}(n, k)$. We will denote the limiting case of $\ell_{m}^{(r)}(n, k)$ as $m \rightarrow \infty$ (in particular, if $m>n-k$ ) by $\ell^{(r)}(n, k)$. Then $\ell^{(r)}(n, k)$ counts the set of all $r$-Lah distributions of size $n+r$ having $k+r$ blocks (which will be denoted by $\left.\mathcal{L}^{(r)}(n, k)\right)$, as there is no restriction on the block cardinalities when $m>n-k$. When $r=0$ and $m>n-k$, members of $\mathcal{L}_{m}^{(r)}(n, k)$ coincide with the usual Lah distributions enumerated by [19, Sequence A008297] as there is no restriction also on block membership.

The recurrences below will be based on the following initial conditions. First, let $\ell_{m}^{(r)}(n, k)=0$ if any of the parameters are negative or if $0 \leq n<k$. If $m=0$, then we will assume $\ell_{0}^{(r)}(n, k)=0$ for all $n$ and $k$ if $r>0$, or if $r=0$ and $n$ and $k$ are not both zero, with $\ell_{0}^{(0)}(0,0)=1$. If $r=0$, then an inclusion-exclusion argument gives

$$
\ell_{m}^{(0)}(n, k)=\frac{n !}{k !} \sum_{i=0}^{\left\lfloor\frac{n-1}{m}\right\rfloor}(-1)^{i}\left(\begin{array}{c}
k \\
i
\end{array}\right)\left(\begin{array}{c}
n-m i-1 \\
k-1
\end{array}\right), \quad n, k, m \geq 1,
$$

with $\ell_{m}^{(0)}(n, 0)=\delta_{n, 0}$ for all $m \geq 1$. If $n=0$, then $\ell_{m}^{(r)}(0, k)=\delta_{k, 0}$ for all $m, r \geq 1$. Furthermore, the factorial of a negative number will always be taken to be 1 for convenience and the binomial coefficient $\left(\begin{array}{l}n \\ k\end{array}\right)$ will be assumed to be zero if $n$ or $k$ is negative or if $k>n \geq 0$.

We now give perhaps the simplest recurrence satisfied by the $\ell_{m}^{(r)}(n, k)$.

Proposition 2.1. If $n, m \geq 1$ and $k, r \geq 0$, then

$$
\begin{aligned}
k \ell_{m}^{(r)}(n, k)= & n k \ell_{m}^{(r)}(n-1, k)+n \ell_{m}^{(r)}(n-1, k-1) \\
& -\frac{n !}{(n-m-1) !} \ell_{m}^{(r)}(n-m-1, k-1) .
\end{aligned}
$$

Proof. Note that we may assume $k \geq 1$ in (2.1), for it clearly holds if $k=0$. The left side of (2.1) then counts all "marked" members of $\mathcal{L}_{m}^{(r)}(n, k)$ wherein one of the non-special blocks is marked. Alternatively, in the case when the marked non-special block is not a singleton, one can set aside an element of $[r+1, r+n]$ and then add it at the beginning of the block that is marked within a member of $\mathcal{L}_{m}^{(r)}(n-1, k)$, yielding $n k \ell_{m}^{(r)}(n-1, k)$ possibilities. However, one would need to subtract $(m+1) !\left(\begin{array}{c}n \\ m+1\end{array}\right) \ell_{m}^{(r)}(n-m-1, k-1)$ which accounts for the case when adding the extra element results in a block of size $m+1$. On the other hand, if the marked non-special block is a singleton, then there are $n \ell_{m}^{(r)}(n-1, k-1)$ possibilities, and combining this case with the prior gives (2.1).

Remark 2.2. The case of (2.1) when $r=0$ and $m \rightarrow \infty$ is given in [18, Formula $3.5]$, where a $q$-generalization in terms of a statistic on Laguerre configurations is provided. 
We observe now the following further special values of $\ell_{m}^{(r)}(n, k)$. If $m=1$, then $\ell_{1}^{(r)}(n, k)=\delta_{n, k}$ for all $n, k, r \geq 0$, so it will often be assumed in proofs that $m \geq 2$. If $k=n$, then we have $\ell_{m}^{(r)}(n, n)=1$ for all $m \geq 1, r \geq 0$. If $k=n-1$ where $n \geq 1$, then considering whether a special or a non-special block has cardinality two implies $\ell_{m}^{(r)}(n, n-1)=n(2 r+n-1)$ if $m \geq 2$. Finally, if $k=n-2$ where $n \geq 2$, then considering several cases concerning the blocks that are not singletons gives the formula

$$
\ell_{m}^{(r)}(n, n-2)= \begin{cases}2 r(2 r+1)\left(\begin{array}{l}
n \\
2
\end{array}\right)+6(2 r+1)\left(\begin{array}{l}
n \\
3
\end{array}\right)+12\left(\begin{array}{c}
n \\
4
\end{array}\right), & \text { if } m \geq 3 \\
4 r(r-1)\left(\begin{array}{l}
n \\
2
\end{array}\right)+12 r\left(\begin{array}{l}
n \\
3
\end{array}\right)+12\left(\begin{array}{c}
n \\
4
\end{array}\right), & \text { if } m=2\end{cases}
$$

\section{Properties of restricted $r$-Lah numbers}

The $\ell_{m}^{(r)}(n, k)$ are also defined by the following recurrences, where $m$ and/or $r$ is changing as well.

Proposition 3.1. If $n, m \geq 1$ and $k, r \geq 0$, then

$$
\begin{aligned}
\ell_{m}^{(r)}(n, k)= & \ell_{m}^{(r)}(n-1, k-1)+(n-1+k+2 r) \ell_{m}^{(r)}(n-1, k) \\
& -(m+1) !\left(\begin{array}{c}
n-1 \\
m
\end{array}\right) \ell_{m}^{(r)}(n-m-1, k-1) \\
& -r(m+1) !\left(\begin{array}{c}
n-1 \\
m-1
\end{array}\right) \ell_{m}^{(r-1)}(n-m, k)
\end{aligned}
$$

and

$$
\begin{aligned}
& \ell_{m}^{(r)}(n, k) \\
& =n ! \sum_{i=0}^{k} \sum_{j=0}^{r} \frac{m^{j}}{i !(n-m i-(m-1) j) !}\left(\begin{array}{l}
r \\
j
\end{array}\right) \ell_{m-1}^{(r-j)}(n-m i-(m-1) j, k-i) .
\end{aligned}
$$

Proof. To show (3.1), first observe that there are $\ell_{m}^{(r)}(n-1, k-1)$ members of $\mathcal{L}_{m}^{(r)}(n, k)$ such that $n+r$ comprises its own block. Otherwise, the element $n+r$ directly follows some member of $[n+r-1]$ or occurs at the very beginning of a block containing at least one other element. This yields altogether $(n-1+k+$ $2 r) \ell_{m}^{(r)}(n-1, k)$ possibilities if the block sizes were unrestricted. However, one needs to subtract $(m+1) !\left(\begin{array}{c}n-1 \\ m\end{array}\right) \ell_{m}^{(r)}(n-m-1, k-1)$ and also $r(m+1) !\left(\begin{array}{c}n-1 \\ m-1\end{array}\right) \ell_{m}^{(r-1)}(n-m, k)$ to account for the cases when $n+r$ is added, respectively, to a non-special or special block already containing $m$ elements. Combining the previous cases then gives (3.1).

To show (3.2), let $i$ and $j$ denote, respectively, the number of non-special and special blocks of size $m$ within $\lambda \in \mathcal{L}_{m}^{(r)}(n, k)$. Then there are

$$
\frac{1}{i !}\left(\begin{array}{c}
n \\
m, \ldots, m, m-1, \ldots, m-1, n-m i-(m-1) j
\end{array}\right)(m !)^{i+j}
$$




$$
=\frac{n ! m^{j}}{i !(n-m i-(m-1) j) !}
$$

ways in which to choose and order the elements occupying these blocks of $\lambda$, where it is assumed that $m i+(m-1) j \leq n$, and $\left(\begin{array}{l}r \\ j\end{array}\right)$ choices for the special blocks that are to contain $m$ elements. The rest of the blocks of $\lambda$ all have size at most $m-1$ and hence there are $\ell_{m-1}^{(r-j)}(n-m i-(m-1) j, k-i)$ ways to arrange the remaining elements of $[n+r]$. Considering all possible $i$ and $j$ gives (3.2).

Remark 3.2. Letting $m>n$ in (3.1) gives [16, Theorem 3.1], while letting $r=0$ and $m>n$ in (3.2) gives a Lah analogue of the Stirling number identity found in [11, Proposition 3].

From (3.1), we get the following identity for $n>k \geq 0$ :

$$
\begin{aligned}
\ell_{m}^{(r)}(n, k)= & \sum_{i=0}^{k}(n+k+2 r-2 i-1) \ell_{m}^{(r)}(n-i-1, k-i) \\
& -(m+1) ! \sum_{i=0}^{k}\left(\left(\begin{array}{c}
n-i-1 \\
m
\end{array}\right) \ell_{m}^{(r)}(n-m-i-1, k-i-1)\right. \\
& \left.+r\left(\begin{array}{c}
n-i-1 \\
m-1
\end{array}\right) \ell_{m}^{(r-1)}(n-m-i, k-i)\right) .
\end{aligned}
$$

To show (3.3), one can induct on $k$ (starting with $k=0$ ) and use (3.1) to show that the $(n-1, k)$ case of the identity implies the $(n, k+1)$ case for all $n$ and $k$.

Theorem 3.3. If $n \geq 1, m \geq 2$ and $k, r \geq 0$, then

$$
\begin{aligned}
\ell_{m}^{(r)}(n, k)=\frac{n !}{k !} \sum_{i=0}^{r} \sum_{j=0}^{i} \sum_{s=0}^{k} & \frac{(k-s+j) !}{(n-m s-r+i) !}\left(\begin{array}{c}
k \\
s
\end{array}\right)\left(\begin{array}{c}
r \\
j, i-j, r-i
\end{array}\right) \\
& \times \ell_{m-1}^{(r-i)}(n-m s-r+i, k-s+j) .
\end{aligned}
$$

Proof. To enumerate the members of $\mathcal{L}_{m}^{(r)}(n, k)$, first let $i$ denote the number of special elements that start their respective blocks and let $s$ be the number of nonspecial blocks of size $m$. Then there are $\left(\begin{array}{c}n \\ m, \ldots, m, n-m s\end{array}\right) \frac{(m !)^{s}}{s !}$ ways in which to select and order the elements within these non-special blocks. Next, let $j$ denote the number of special elements that start non-singleton blocks, where $0 \leq j \leq i$. Now choose $i$ members of $[r]$ to start blocks and from these select $j$ that are not to form singleton blocks, which can be done in $\left(\begin{array}{l}r \\ i\end{array}\right)\left(\begin{array}{l}i \\ j\end{array}\right)$ ways (note that the other $i-j$ members of $[r]$ are to occur as singletons). Concerning the remaining $r-i$ members of $[r]$ which do not start blocks, we pick "predecessor" elements from the remaining $n-m s$ members of $[r+1, r+n]$, which can be done in $(n-m s) \frac{r-i}{}$ ways, where it is implicit that $s \leq \min \{k,\lfloor n / m\rfloor\}$.

At this point, we treat each of these $r-i$ special elements, together with their predecessors, as single (special) elements. We form a partition of these elements, 
together with the $n-m s-r+i$ remaining non-special elements, in which there are $k-s+j$ non-special blocks and all blocks are of size less than $m$, which can be effected in $\ell_{m-1}^{(r-i)}(n-m s-r+i, k-s+j)$ ways. Let $\lambda$ denote one of the resulting partitions. Then we choose $j$ of the non-special blocks of $\lambda$ and add, one-per-block, the $j$ special elements that were selected earlier to start non-singleton special blocks, which can be done in $\left(\begin{array}{c}k-s+j \\ j\end{array}\right) j$ ! ways. We leave unchanged the remaining blocks of $\lambda$. Note that all of the special blocks in the resulting partition $\rho$ actually can have size up to $m$ due to the addition of these elements and to the occurrence of the "double" special elements described above, and thus $\rho \in \mathcal{L}_{m}^{(r-i+j)}(n-m s, k-s)$ (after standardization). Observe that the non-special blocks of $\rho$ are each of size at most $m-1$ since they correspond to the $k-s$ unselected non-special blocks of $\lambda$. Adding the $i-j$ singleton special blocks from above, and also the $s$ non-special blocks of size $m$, to $\rho$ yields an enumerated member of $\mathcal{L}_{m}^{(r)}(n, k)$ for the given $i, j$ and $s$. Note that all members of $\mathcal{L}_{m}^{(r)}(n, k)$ arise uniquely in this way as $i, j$ and $s$ vary.

Summing over these parameters then implies

$$
\begin{aligned}
& \ell_{m}^{(r)}(n, k)=\sum_{i=0}^{r} \sum_{j=0}^{i} \sum_{s=0}^{k}\left(\begin{array}{l}
r \\
i
\end{array}\right)\left(\begin{array}{l}
i \\
j
\end{array}\right)\left(\begin{array}{c}
n \\
m, \ldots, m, n-m s
\end{array}\right) \frac{(m !)^{s}}{s !}(n-m s) \frac{r-i}{k-s+j}\left(\begin{array}{c}
k \\
j
\end{array}\right) j ! \\
& \times \ell_{m-1}^{(r-i)}(n-m s-r+i, k-s+j) \\
& =\sum_{i=0}^{r} \sum_{j=0}^{i} \sum_{s=0}^{k} \frac{n ! r !}{(r-i) ! j !(i-j) ! s !} \cdot \frac{(n-m s) \frac{r-i}{}}{(n-m s) !} \cdot \frac{(k-s+j) !}{(k-s) !} \\
& \times \ell_{m-1}^{(r-i)}(n-m s-r+i, k-s+j) \\
& =\frac{n !}{k !} \sum_{i=0}^{r} \sum_{j=0}^{i} \sum_{s=0}^{k} \frac{(k-s+j) !}{(n-m s-r+i) !}\left(\begin{array}{l}
k \\
s
\end{array}\right)\left(\begin{array}{c}
r \\
j, i-j, r-i
\end{array}\right) \\
& \times \ell_{m-1}^{(r-i)}(n-m s-r+i, k-s+j),
\end{aligned}
$$

as desired.

Remark 3.4. Letting $m$ be large in (3.4) gives the following apparently new identity for the $r$-Lah number $\ell^{(r)}(n, k)$ :

$$
\ell^{(r)}(n, k)=\frac{n !}{k !} \sum_{i=0}^{r} \sum_{j=0}^{i} \frac{(k+j) !}{(n-r+i) !}\left(\begin{array}{c}
r \\
j, i-j, r-i
\end{array}\right) \ell^{(r-i)}(n-r+i, k+j) .
$$

Considering whether or not a member of $\mathcal{L}_{m}^{(r)}(n, k)$ contains any special or nonspecial singleton blocks leads to the following further recurrences.

Theorem 3.5. If $n, k \geq 0, r \geq 1$ and $m \geq 2$, then

$$
\ell_{m}^{(r)}(n, k)=\sum_{i=1}^{r}(-1)^{i-1}\left(\begin{array}{l}
r \\
i
\end{array}\right) \ell_{m}^{(r-i)}(n, k)
$$




$$
+\frac{n !}{k !} \sum_{i=0}^{r} \sum_{s=0}^{k} \frac{(k-s+i) !}{(n-m s-r+i) !}\left(\begin{array}{l}
r \\
i
\end{array}\right)\left(\begin{array}{l}
k \\
s
\end{array}\right) \ell_{m-1}^{(r-i)}(n-m s-r+i, k-s+i)
$$

and

$$
\begin{aligned}
& \ell_{m}^{(r)}(n, k)=\sum_{i=1}^{k}(-1)^{i-1}\left(\begin{array}{c}
n \\
i
\end{array}\right) \ell_{m}^{(r)}(n-i, k-i) \\
& \quad+n ! \sum_{i=0}^{r} \frac{m^{i}}{(n-k-(m-1) i) !}\left(\begin{array}{c}
r \\
i
\end{array}\right) \ell_{m-1}^{(r-i)}(n-k-(m-1) i, k) .
\end{aligned}
$$

Proof. We will assume $n, k \geq 1$, as the formulas will be seen to hold also in the case when $n$ or $k$ is zero. To show (3.6), first note that letting $i=j$ in the proof of (3.4) above gives the cardinality of all members of $\mathcal{L}_{m}^{(r)}(n, k)$ in which no element of $[r]$ forms its own block and that the double sum expression on the right side of (3.6) corresponds to taking only the $j=i$ term in the $j$-indexed sum in (3.4). Let $\widetilde{\mathcal{L}}_{m}^{(r)}(n, k)$ denote the subset of $\mathcal{L}_{m}^{(r)}(n, k)$ whose members contain at least one special singleton block. By subtraction, the difference $\ell_{m}^{(r)}(n, k)-\left|\widetilde{\mathcal{L}}_{m}^{(r)}(n, k)\right|$ gives the cardinality of all members of $\mathcal{L}_{m}^{(r)}(n, k)$ containing no special singleton blocks. On the other hand, by the principle of inclusion-exclusion, this cardinality is also given by

$$
\sum_{i=0}^{r}(-1)^{i}\left(\begin{array}{l}
r \\
i
\end{array}\right) \ell_{m}^{(r-i)}(n, k)=\ell_{m}^{(r)}(n, k)-\sum_{i=1}^{r}(-1)^{i-1}\left(\begin{array}{l}
r \\
i
\end{array}\right) \ell_{m}^{(r-i)}(n, k) .
$$

Comparing expressions gives $\left|\widetilde{\mathcal{L}}_{m}^{(r)}(n, k)\right|=\sum_{i=1}^{r}(-1)^{i-1}\left(\begin{array}{c}r \\ i\end{array}\right) \ell_{m}^{(r-i)}(n, k)$, which implies (3.6).

To show (3.7), note that by similar reasoning, the first sum on the right-hand side counts all members of $\mathcal{L}_{m}^{(r)}(n, k)$ containing at least one non-special singleton block. To enumerate those $\lambda \in \mathcal{L}_{m}^{(r)}(n, k)$ that do not contain any, first suppose that exactly $i$ of the special blocks of $\lambda$ have size $m$. Then there are $\left(\begin{array}{c}r \\ i\end{array}\right)\left(\begin{array}{c}n \\ m-1, \ldots, m-1, n-(m-1) i\end{array}\right)(m !)^{i}=\frac{n !}{(n-(m-1) i) !}\left(\begin{array}{c}r \\ i\end{array}\right) m^{i}$ ways to select and order the elements that comprise these blocks of $\lambda$, where $i \leq\lfloor n /(m-1)\rfloor$. Next, we pick $k$ of the remaining elements of $[r+1, r+n]$, which can be done in $\left(\begin{array}{c}n-(m-1) i \\ k\end{array}\right)$ ways, and set them aside. We then arrange the rest of the $n-k-(m-1) i$ elements of $[r+1, r+n]$, together with the $r-i$ unchosen elements of $[r]$, according to a member of $\mathcal{L}_{m-1}^{(r-i)}(n-k-(m-1) i, k)$. Then we add the $k$ non-special elements that were set aside to the beginning of the non-special blocks of this partition, one-per-block, according to an arbitrary permutation of $[k]$, to obtain $\lambda$. Thus, we get

$$
\begin{aligned}
& n ! \sum_{i=0}^{r} \frac{m^{i}}{(n-(m-1) i) !}\left(\begin{array}{c}
r \\
i
\end{array}\right)\left(\begin{array}{c}
n-(m-1) i \\
k
\end{array}\right) k ! \ell_{m-1}^{(r-i)}(n-k-(m-1) i, k) \\
& =n ! \sum_{i=0}^{r} \frac{m^{i}}{(n-k-(m-1) i) !}\left(\begin{array}{c}
r \\
i
\end{array}\right) \ell_{m-1}^{(r-i)}(n-k-(m-1) i, k)
\end{aligned}
$$


members of $\mathcal{L}_{m}^{(r)}(n, k)$ that do not contain a non-special singleton block, which gives (3.7).

We were unable to find in the literature the $r$-Lah identities corresponding to the limiting cases of (3.6) and (3.7) as $m \rightarrow \infty$. Before stating the next result, let

$$
L_{m}^{(r)}(n, k)=\sum_{j=k}^{n}\left(\begin{array}{l}
j \\
k
\end{array}\right) \ell_{m}^{(r)}(n, j) \quad \text { and } \quad x^{\bar{k}}=x(x+1) \cdots(x+k-1),
$$

for a variable $x$. We have the following relationship between $\ell_{m}^{(r)}(n, k)$ and its upper binomial transform $L_{m}^{(r)}(n, k)$.

Theorem 3.6. If $n, k, m, p \geq 1$ and $r \geq 0$, then

$$
\sum_{j=1}^{n}(j+p+2 r)^{\bar{k}} \ell_{m}^{(r)}(n, j)=\sum_{i=0}^{n} \sum_{s=0}^{p}(i+s) !\left(\begin{array}{c}
p \\
s
\end{array}\right) \ell^{(r)}(k, i+s) L_{m}^{(r)}(n, i) .
$$

Proof. First note that $(j+p+2 r)^{\bar{k}}$ counts partitions of $[k]$ into $j+p+2 r$ labeled, contents-ordered blocks in which some of the blocks may be empty. Let $\mathcal{A}_{n, k}^{(p)}$ denote the set of ordered pairs $(\alpha, \beta)$ in which $\alpha \in \mathcal{L}_{m}^{(r)}(n, j)$ and $\beta$ is a partition enumerated by $(j+p+2 r)^{\bar{k}}$ for some $1 \leq j \leq n$. Then $\left|\mathcal{A}_{n, k}^{(p)}\right|$ is given by the lefthand side of (3.8). Let $\mathcal{B}_{n, k}^{(p)}$ denote the set of triples $(\gamma, \delta, \epsilon)$ such that $\gamma \in \mathcal{L}_{m}^{(r)}(n, j)$, where $i$ of the non-special blocks of $\gamma$ are circled for some $0 \leq i \leq j \leq n, \delta$ is a subset of $[p]$ of size $s$ for some $s$, and $\epsilon$ is a member of $\mathcal{L}^{(r)}(k, i+s)$ in which the non-special blocks can occur in any order. Then $\left|\mathcal{B}_{n, k}^{(p)}\right|$ is given by

$$
\sum_{j=1}^{n} \sum_{s=0}^{p} \sum_{i=0}^{j}(i+s) !\left(\begin{array}{l}
j \\
i
\end{array}\right)\left(\begin{array}{l}
p \\
s
\end{array}\right) \ell^{(r)}(k, i+s) \ell_{m}^{(r)}(n, j),
$$

which can be rewritten to give the right-hand side of (3.8).

To complete the proof, we define a bijection between the sets $\mathcal{A}_{n, k}^{(p)}$ and $\mathcal{B}_{n, k}^{(p)}$. To do so, let $(\alpha, \beta) \in \mathcal{A}_{n, k}^{(p)}$ and we construct a member of $\mathcal{B}_{n, k}^{(p)}$. Consider the labels of the non-empty blocks among the first $j$ blocks of $\beta$ (starting from the left) and then those among the non-empty of the next $p$ blocks of $\beta$. This determines (possibly empty) subsets $S_{1}$ and $S_{2}$ of $[j]$ and $[p]$, respectively. Let $\delta=S_{2}$ and $\gamma$ be obtained from $\alpha$ by circling the non-special blocks of $\alpha$ corresponding to the subset $S_{1}$, where we assume that the non-special blocks of $\gamma$ are arranged left-to-right in increasing order of smallest elements. To form $\epsilon$, we first create its non-special blocks using the non-empty blocks among the first $j+p$ blocks of $\beta$ where each element of $\beta$ is increased by $r$ (note that all of $\beta$ 's blocks are labeled in increasing order from left to right, including the empty ones). To create the $q$-th special block of $\epsilon$ where $1 \leq q \leq r$, we form the word $\rho_{1} q \rho_{2}$, where $\rho_{1}$ and $\rho_{2}$ denote respectively the ordered contents of the $(j+p+2 q-1)$-st and $(j+p+2 q)$-th blocks of $\beta$ (and $\rho_{1}$ and $\rho_{2}$ 
are represented using letters in $[r+1, r+k])$. Note that there is no restriction on the block cardinalities of $\epsilon$ and that the non-special blocks of $\epsilon$ are themselves ordered (since the blocks of $\beta$ were labeled), whence there are $(i+s) ! \ell^{(r)}(k, i+s)$ possibilities for $\epsilon$ where $i=\left|S_{1}\right|$ and $s=\left|S_{2}\right|$. One may verify that the mapping $(\alpha, \beta) \mapsto(\gamma, \delta, \epsilon)$ defined by the above construction is a bijection between $\mathcal{A}_{n, k}^{(p)}$ and $\mathcal{B}_{n, k}^{(p)}$, which completes the proof.

We have the following further $r$-dependent recurrence.

Theorem 3.7. If $n, k \geq 0, m \geq 1$ and $1 \leq s \leq r$, then

$$
\begin{aligned}
\ell_{m}^{(r)}(n, k)=n ! \sum_{i=0}^{(m-1) s} \frac{\ell_{m}^{(r-s)}(n-i, k)}{(n-i) !} & {\left[\sum_{j=0}^{\left\lfloor\frac{i}{m}\right\rfloor} \sum_{p=0}^{j}(-1)^{j}\left(\begin{array}{c}
s \\
p, j-p, s-j
\end{array}\right)\right.} \\
& \left.\times\left(\begin{array}{c}
i+2 s-(m+1) j-1 \\
2 s-p-1
\end{array}\right)(m+1)^{p}\right] .
\end{aligned}
$$

Proof. We start by considering the number $t$ of elements in the first $s$ special blocks within a member of $\mathcal{L}_{m}^{(r)}(n, k)$, where $s \leq t \leq m s$. Then there are $\left(\begin{array}{c}n \\ t-s\end{array}\right)$ choices for the non-special elements within these blocks and $\ell_{m}^{(r-s)}(n-t+s, k)$ ways in which to arrange elements within the non-special and the final $r-s$ special blocks. Finally, there are

$$
(t-s) ! \sum_{\substack{\lambda_{1}+\cdots+\lambda_{s}=t \\ 1 \leq \lambda_{i} \leq m}} \lambda_{1} \cdots \lambda_{s}
$$

ways in which to arrange the elements in the first $s$ special blocks. To realize this, note that the multi-indexed sum counts all compositions of $t$ with $s$ parts, where the $i$-th part for each $i$ has size $\lambda_{i}, 1 \leq \lambda_{i} \leq m$, and is colored in one of $\lambda_{i}$ ways. The $i$-th special block for each $i \in[s]$ is then to have cardinality $\lambda_{i}$, within which $i$ is to occupy the $b_{i}$-th position from the left, where $b_{i}$ denotes the color assigned to the part $\lambda_{i}$. The $t-s$ non-special elements within the first $s$ special blocks can occur in any order in a left-to-right scan of their contents, which accounts for the $(t-s)$ ! factor. Combining the above observations gives

$$
\ell_{m}^{(r)}(n, k)=\sum_{t=s}^{m s}\left(\sum_{\substack{\lambda_{1}+\cdots+\lambda_{s}=t \\ 1 \leq \lambda_{i} \leq m}} \lambda_{1} \cdots \lambda_{s}\right) \frac{n !}{(n-t+s) !} \ell_{m}^{(r-s)}(n-t+s, k) .
$$

We now simplify the multi-sum in (3.10). To do so, we make use of the inclusionexclusion principle and sieve out from the set of all (colored) compositions of $t$ having $s$ parts those whose parts are at most $m$. Consider the number $j$ of parts of size exceeding $m$; note that $(m+1) j+(s-j) \leq t$ gives $j \leq\left\lfloor\frac{t-s}{m}\right\rfloor$, which we denote by $u$. Then $t \leq m s$ implies $u \leq s$. Thus, we have

$$
\sum_{\substack{\lambda_{1}+\cdots+\lambda_{s}=t \\ 1 \leq \lambda_{i} \leq m}} \lambda_{1} \cdots \lambda_{s}
$$




$$
\begin{aligned}
& =\sum_{j=0}^{u}(-1)^{j}\left(\begin{array}{l}
s \\
j
\end{array}\right) \sum_{\substack{\lambda_{1}+\cdots+\lambda_{s}=t-(m+1) j \\
\lambda_{i} \geq 0}}\left(\lambda_{1}+m+1\right) \cdots\left(\lambda_{j}+m+1\right) \lambda_{j+1} \cdots \lambda_{s} \\
& =\sum_{j=0}^{u}(-1)^{j}\left(\begin{array}{l}
s \\
j
\end{array}\right) \sum_{p=0}^{j}\left(\begin{array}{l}
j \\
p
\end{array}\right)(m+1)^{p} \sum_{\substack{\lambda_{1}+\cdots+\lambda_{s}=t-(m+1) j \\
\lambda_{i} \geq 0}} \lambda_{p+1} \cdots \lambda_{s} \\
& =\sum_{j=0}^{u}(-1)^{j}\left(\begin{array}{l}
s \\
j
\end{array}\right) \sum_{p=0}^{j}\left(\begin{array}{l}
j \\
p
\end{array}\right)(m+1)^{p}\left(\begin{array}{c}
s+t-(m+1) j-1 \\
2 s-p-1
\end{array}\right),
\end{aligned}
$$

where we have used [2, Formula 26] in the last equality. Substituting this into (3.10), and replacing $t$ with $i+s$, gives (3.9).

When $m$ is large in (3.9), note that $j=p=0$ is required in the two inner sums, which yields the following recurrence for $\ell^{(r)}(n, k)$.

Corollary 3.8 (Nyul and Rácz [16]). If $n, k \geq 0$ and $1 \leq s \leq r$, then

$$
\ell^{(r)}(n, k)=\sum_{i=0}^{n-k}(2 s)^{\bar{i}}\left(\begin{array}{c}
n \\
i
\end{array}\right) \ell^{(r-s)}(n-i, k) .
$$

We conclude this section with the following recurrences which are obtained by considering the nature of the singleton blocks within a member of $\mathcal{L}_{m}^{(r)}(n, k)$.

Theorem 3.9. If $n, k \geq 0, m \geq 1$ and $r \geq 0$, then

$$
\ell_{m}^{(r)}(n, k)=\ell_{m}^{(0)}(n, k)+\sum_{j=1}^{r} \sum_{i=1}^{m-1}(i+1) !\left(\begin{array}{c}
n \\
i
\end{array}\right) \ell_{m}^{(r-j)}(n-i, k)
$$

and

$$
\begin{aligned}
& \ell_{m}^{(r)}(n, k)=\sum_{j=0}^{r} \sum_{i=0}^{k} \sum_{t=0}^{j} \frac{(n-k+i) !(i+1)^{\bar{t}}}{(n-k-j+t) !}\left(\begin{array}{c}
n \\
k-i
\end{array}\right)\left(\begin{array}{c}
r \\
t, j-t, r-j
\end{array}\right) \\
& \times \ell_{m-1}^{(j-t)}(n-k-j+t, i+t) .
\end{aligned}
$$

Proof. To show (3.12), consider within a member of $\mathcal{L}_{m}^{(r)}(n, k)$ the smallest $j \in[r]$ if it exists such that the singleton block $\{j\}$ does not occur (note that there are $\ell_{m}^{(0)}(n, k)$ possibilities if no such $j$ exists). If $i+1$ denotes the cardinality of the block containing $j$, where $1 \leq i \leq \min \{m-1, n-k\}$, then there are $\left(\begin{array}{c}n \\ i\end{array}\right)(i+1)$ ! ways in which to select and order the elements belonging to this block. There are thus $\ell_{m}^{(r-j)}(n-i, k)$ ways in which to arrange the remaining $r-j$ special and $n-i$ non-special elements. Considering all $i$ and $j$ gives (3.12).

To show (3.13), first note that we may assume $m \geq 2$ since the formula is seen to hold when $m=1$. Let $r-j$ and $k-i$ denote the number of special and non-special 
singleton blocks, respectively, within a member of $\mathcal{L}_{m}^{(r)}(n, k)$, where $0 \leq j \leq r$ and $0 \leq i \leq k$. We select the elements comprising these blocks in $\left(\begin{array}{c}r \\ j\end{array}\right)\left(\begin{array}{c}n \\ k-i\end{array}\right)$ ways and set them aside. At this point, let us refer to non-singleton special blocks that are to start with a special (non-special, resp.) element as being of type 1 (of type 2, resp.), with type 3 referring to non-singleton non-special blocks. Let $t$ denote the number of type 1 blocks, where $0 \leq t \leq j$. The special elements in these blocks may be chosen in $\left(\begin{array}{l}j \\ t\end{array}\right)$ ways, the set of which we denote by $S$. We now construct $\lambda \in \mathcal{L}_{m}^{(r)}(n, k)$ meeting the above specifications with respect to $i, j$ and $t$. To do so, we first choose $i+j-t$ of the remaining elements of $[r+1, r+n]$ that are to start either one of the $j-t$ blocks of $\lambda$ of type 2 or one of its $i$ blocks of type 3 , which can be done in $\left(\begin{array}{c}n-k+i \\ i+j-t\end{array}\right)$ ways, the set of which we denote by $T$. We place the elements of $T$ aside and then arrange all elements of $[n+r]$ not chosen thus far according to some partition $\rho$, where $\rho$ (when standardized) belongs to $\mathcal{L}_{m-1}^{(j-t)}(n-k-j+t, i+t)$.

We now choose $t$ of the non-special blocks of $\rho$ in one of $\left(\begin{array}{c}i+t \\ t\end{array}\right)$ ways and then add a member of $S$ to the beginning of each of these blocks according to any permutation of $S$. This produces the $t$ type 1 blocks of $\lambda$. Next, we add the elements of $T$, one-per-block, to the beginning of the remaining $i+j-t$ blocks of $\rho$ (i.e., those that did not receive an element of $S$ ), which can be done in $(i+j-t)$ ! ways. This gives all of the blocks of $\lambda$ of type 2 or 3 . Appending as singleton blocks the $r-j$ special and the $k-i$ non-special elements set aside above completes the construction of the enumerated partition $\lambda$. One may verify that all $\lambda$ satisfying the given requirements arise uniquely in this manner. By the preceding, the cardinality of such $\lambda$ is given by

$$
\begin{array}{r}
\left(\begin{array}{c}
r \\
j
\end{array}\right)\left(\begin{array}{c}
n \\
k-i
\end{array}\right)\left(\begin{array}{l}
j \\
t
\end{array}\right)\left(\begin{array}{c}
n-k+i \\
i+j-t
\end{array}\right)\left(\begin{array}{c}
i+t \\
t
\end{array}\right) t !(i+j-t) ! \ell_{m-1}^{(j-t)}(n-k-j+t, i+t) \\
=\frac{(n-k+i) !(i+1)^{\bar{t}}}{(n-k-j+t) !}\left(\begin{array}{c}
n \\
k-i
\end{array}\right)\left(\begin{array}{c}
r \\
t, j-t, r-j
\end{array}\right) \ell_{m-1}^{(j-t)}(n-k-j+t, i+t) .
\end{array}
$$

Summing over $i, j$ and $t$ yields all members of $\mathcal{L}_{m}^{(r)}(n, k)$.

Remark 3.10. Letting $m \rightarrow \infty$ in (3.12) and (3.13) gives further identities for the $r$-Lah numbers. Letting $r=0$ in the second of these identities implies

$$
\ell^{(0)}(n, k)=\sum_{i=0}^{k} \frac{(n-k+i) !}{(n-k) !}\left(\begin{array}{c}
n \\
k-i
\end{array}\right) \ell^{(0)}(n-k, i), \quad n, k \geq 0
$$

which can also be shown directly using the $r=0$ case of (4.4) below. Note that by using the formula from (4.4) in the limiting case of (3.13), one obtains an interesting family of binomial coefficient identities indexed by $r$. 


\section{Explicit formula for $\ell_{m}^{(r)}(n, k)$}

We provide a combinatorial proof of the following expression for $\ell_{m}^{(r)}(n, k)$ in terms of binomial coefficients.

Theorem 4.1. If $n, k, m \geq 1$ and $r \geq 0$, then

$$
\ell_{m}^{(r)}(n, k)=\frac{n !}{k !} \sum_{i=0}^{k+r} \sum_{t=0}^{r}(-1)^{i}\left(\begin{array}{c}
r \\
t
\end{array}\right)\left(\begin{array}{c}
k+r-t \\
i-t
\end{array}\right)\left(\begin{array}{c}
n+2 r-m i-t-1 \\
k+2 r-t-1
\end{array}\right) m^{t}
$$

Proof. Let $C_{n, k, r}^{(m)}$ denote the set of compositions of $n+r$ having $k+r$ parts each of size at most $m$ in which the first $r$ parts are colored such that a part of size $x$ is colored in one of $x$ ways (the remaining $k$ parts are not uncolored). Then we have $\ell_{m}^{(r)}(n, k)=\frac{n !}{k !}\left|C_{n, k, r}^{(m)}\right|$. To see this, given $\lambda=\left(\lambda_{1}, \lambda_{2}, \ldots, \lambda_{r+k}\right) \in C_{n, k, r}^{(m)}$, we distribute the elements of $[n+r]$ in blocks such that $\lambda_{1}, \ldots, \lambda_{r}$ correspond to the cardinalities of the special and $\lambda_{r+1}, \ldots, \lambda_{r+k}$ to the cardinalities of the non-special blocks (written in any order), where the element $i \in[r]$ is to occupy the position $a \in\left[\lambda_{i}\right]$ (from the left) within its block if $a$ is the color assigned to the part $\lambda_{i}$ of $\lambda$. Then there are $n$ ! ways to arrange the elements of $[n+r]$ as described once $\lambda$ is specified, and we divide by $k$ ! since the non-special blocks are themselves not to be ordered.

Next, we determine the cardinality of $C_{n, k, r}^{(m)}$ and first show

$$
\begin{aligned}
\left|C_{n, k, r}^{(m)}\right|=\sum_{i=0}^{k+r} & (-1)^{i} \sum_{j=0}^{i}\left(\begin{array}{c}
r \\
j
\end{array}\right)\left(\begin{array}{c}
k \\
i-j
\end{array}\right) \\
& \times \sum_{\lambda_{1}+\cdots+\lambda_{k+r}=n+r-m i}\left(\lambda_{1}+m\right) \cdots\left(\lambda_{j}+m\right) \lambda_{j+1} \cdots \lambda_{r},
\end{aligned}
$$

where the $\lambda_{i}$ are positive in the innermost sum. To do so, first let $C_{n, k, r}^{*}$ denote the set of compositions of $n+k$ having $k+r$ parts in which the first $r$ parts are colored just as members of $C_{n, k, r}^{(m)}$ were above, where now part sizes are unrestricted and where a (possibly empty) subset of the parts of size $m+1$ or more is circled. Let $C_{n, k, r}^{*}(i)$ denote the subset of $C_{n, k, r}^{*}$ containing exactly $i$ circled parts. Then we have $\left|C_{n, k, r}^{(m)}\right|=\sum_{i=0}^{k+r}(-1)^{i}\left|C_{n, k, r}^{*}(i)\right|$. To see this, let members of $C_{n, k, r}^{*}(i)$ have sign $(-1)^{i}$. Define a sign-changing involution on $\cup_{i=0}^{k+r} C_{n, k, r}^{*}(i)$ by identifying the leftmost part of size greater than $m$ and either circling or uncircling it (where the color is preserved, if the part is among the first $r$ ). The survivors of this involution comprise the set $C_{n, k, r}^{(m)}$, so to complete the proof of (4.2), it suffices to show

$$
\begin{aligned}
& \left|C_{n, k, r}^{*}(i)\right| \\
& =\sum_{j=0}^{i}\left(\begin{array}{c}
r \\
j
\end{array}\right)\left(\begin{array}{c}
k \\
i-j
\end{array}\right) \sum_{\lambda_{1}+\cdots+\lambda_{k+r}=n+r-m i}\left(\lambda_{1}+m\right) \cdots\left(\lambda_{j}+m\right) \lambda_{j+1} \cdots \lambda_{r} .
\end{aligned}
$$


To establish (4.3), consider the number $j$ of parts among the first $r$ that are circled within a member of $C_{n, k, r}^{*}(i)$. Then there are $\left(\begin{array}{l}r \\ j\end{array}\right)\left(\begin{array}{c}k \\ i-j\end{array}\right)$ possible ways to select the parts that are to be circled. Note that the number of possible ways in which to color the first $r$ parts depends on how many and not which of these parts are circled. Thus, once $j$ is given, we may assume that it is the first $j$ parts that are circled. Once the circled parts of $\lambda \in C_{n, k, r}^{*}(i)$ are specified, it follows that there are

$$
\sum_{\lambda_{1}+\cdots+\lambda_{k+r}=n+r-m i}\left(\lambda_{1}+m\right) \cdots\left(\lambda_{j}+m\right) \lambda_{j+1} \cdots \lambda_{r}
$$

ways to determine the sizes of all the parts of $\lambda$ together with the colors of the first $r$ parts. Considering all possible $j$ then implies (4.3) and thus (4.2), as desired.

Now observe that

$$
\begin{aligned}
& \sum_{\substack{\lambda_{1}+\cdots+\lambda_{k+r}=n+r-m i \\
\lambda_{\ell} \geq 1}}\left(\lambda_{1}+m\right) \cdots\left(\lambda_{j}+m\right) \lambda_{j+1} \cdots \lambda_{r} \\
& =\sum_{t=0}^{j}\left(\begin{array}{l}
j \\
t
\end{array}\right) m^{t} \sum_{\substack{\lambda_{1}+\cdots+\lambda_{k+r}=n+r-m i \\
\lambda_{\ell} \geq 1}} \lambda_{t+1} \lambda_{t+2} \cdots \lambda_{r} \\
& =\sum_{t=0}^{j}\left(\begin{array}{l}
j \\
t
\end{array}\right) m^{t} \sum_{\substack{\lambda_{1}+\cdots+\lambda_{k+r}=n-k+r-m i-t \\
\lambda_{t+1}, \ldots, \lambda_{r} \geq 1 \\
\lambda_{\ell} \geq 0 \text { otherwise }}} \lambda_{t+1} \lambda_{t+2} \cdots \lambda_{r} \\
& =\sum_{t=0}^{j}\left(\begin{array}{l}
j \\
t
\end{array}\right)\left(\begin{array}{c}
n+2 r-m i-t-1 \\
k+2 r-t-1
\end{array}\right) m^{t},
\end{aligned}
$$

where we have used [2, Formula 26] in the last equality. Thus, by (4.2) and [7, Identity 5.23], we get

$$
\begin{aligned}
\left|C_{n, k, r}^{(m)}\right| & =\sum_{i=0}^{k+r}(-1)^{i} \sum_{j=0}^{i}\left(\begin{array}{l}
r \\
j
\end{array}\right)\left(\begin{array}{c}
k \\
i-j
\end{array}\right) \sum_{t=0}^{j}\left(\begin{array}{l}
j \\
t
\end{array}\right)\left(\begin{array}{c}
n+2 r-m i-t-1 \\
k+2 r-t-1
\end{array}\right) m^{t} \\
& =\sum_{i=0}^{k+r}(-1)^{i} \sum_{t=0}^{r}\left(\begin{array}{l}
r \\
t
\end{array}\right)\left(\begin{array}{c}
n+2 r-m i-t-1 \\
k+2 r-t-1
\end{array}\right) m^{t} \sum_{j=t}^{i}\left(\begin{array}{c}
k \\
i-j
\end{array}\right)\left(\begin{array}{c}
r-t \\
j-t
\end{array}\right) \\
& =\sum_{i=0}^{k+r}(-1)^{i} \sum_{t=0}^{r}\left(\begin{array}{l}
r \\
t
\end{array}\right)\left(\begin{array}{c}
k+r-t \\
i-t
\end{array}\right)\left(\begin{array}{c}
n+2 r-m i-t-1 \\
k+2 r-t-1
\end{array}\right) m^{t},
\end{aligned}
$$

which implies (4.1).

Allowing $m$ to be large in (4.1) recovers the following explicit formula for $\ell^{(r)}(n, k)$. 
Corollary 4.2 (Nyul and Rácz [16]). If $n, k \geq 1$ and $r \geq 0$, then

$$
\ell^{(r)}(n, k)=\frac{n !}{k !}\left(\begin{array}{c}
n+2 r-1 \\
k+2 r-1
\end{array}\right) .
$$

Let $f_{k, m}^{(r)}(x)=\sum_{n \geq k} \ell_{m}^{(r)}(n, k) \frac{x^{n}}{n !}$. Multiplying both sides of (4.1) by $\frac{x^{n}}{n !}$, summing over $n \geq k$ and interchanging summation, we have

$$
\begin{aligned}
& f_{k, m}^{(r)}(x)=\frac{x^{k}}{k !(1-x)^{k+2 r}} \sum_{i=0}^{k+r} \sum_{t=0}^{i}(-1)^{i}\left(\begin{array}{l}
r \\
t
\end{array}\right)\left(\begin{array}{c}
k+r-t \\
i-t
\end{array}\right) m^{t} x^{m i}(1-x)^{t} \\
& =\frac{x^{k}}{k !(1-x)^{k+2 r}} \sum_{t=0}^{r}\left(\begin{array}{l}
r \\
t
\end{array}\right) m^{t}\left(x^{m+1}-x^{m}\right)^{t} \sum_{i=0}^{k+r-t}(-1)^{i}\left(\begin{array}{c}
k+r-t \\
i
\end{array}\right) x^{m i} \\
& =\frac{x^{k}\left(1-x^{m}\right)^{k+r}}{k !(1-x)^{k+2 r}} \sum_{t=0}^{r}\left(\begin{array}{c}
r \\
t
\end{array}\right)\left[\frac{m x^{m}(x-1)}{1-x^{m}}\right]^{t} \\
& =\frac{\left(x-x^{m+1}\right)^{k}}{k !(1-x)^{k+2 r}}\left(1-(m+1) x^{m}+m x^{m+1}\right)^{r} .
\end{aligned}
$$

Let

$$
f_{m}^{(r)}(x, y)=\sum_{n \geq 0}\left(\sum_{k=0}^{n} \ell_{m}^{(r)}(n, k) y^{k}\right) \frac{x^{n}}{n !} .
$$

Multiplying the last equality by $y^{k}$, and summing over $k \geq 0$, yields the following result.

Corollary 4.3. If $m \geq 1$ and $r \geq 0$, then

$$
f_{m}^{(r)}(x, y)=\left(\frac{1-(m+1) x^{m}+m x^{m+1}}{(1-x)^{2}}\right)^{r} \exp \left(\frac{x\left(1-x^{m}\right)}{1-x} y\right) .
$$

Consider $L_{n, m}^{(r)}$ defined by

$$
L_{n, m}^{(r)}=\sum_{k=0}^{n} \ell_{m}^{(r)}(n, k) \frac{(-1)^{n-k}}{k+1}, \quad m \geq 1, r \geq 0 .
$$

Note that when $r=0$, the $L_{n, m}^{(r)}$ provide a Lah analogue to the restricted Cauchy numbers studied in [11], which reduce to the classical Cauchy numbers as $m \rightarrow \infty$. Let $L_{m}^{(r)}(x)=\sum_{n \geq 0} L_{n, m}^{(r)} \frac{x^{n}}{n !}$. Our next result in the $r=0$ case is analogous to the one from [11] for restricted Cauchy numbers.

Proposition 4.4. If $m \geq 1$ and $r \geq 0$, then

$$
L_{m}^{(r)}(-x)=\frac{\left(1-(m+1) x^{m}+m x^{m+1}\right)^{r}\left(1-\exp \left(\frac{x\left(1-x^{m}\right)}{x-1}\right)\right)}{x\left(1-x^{m}\right)(1-x)^{2 r-1}} .
$$


Proof. Multiplying both sides of (4.6) by $\frac{(-x)^{n}}{n !}$, and summing over $n \geq 0$, implies

$$
\begin{aligned}
L_{m}^{(r)}(-x) & =\sum_{k \geq 0} \frac{(-1)^{k} f_{k, m}^{(r)}(x)}{k+1}=\int_{0}^{1} \sum_{k \geq 0} f_{k, m}^{(r)}(x)(-y)^{k} d y=\int_{0}^{1} f_{m}^{(r)}(x,-y) d y \\
& =\frac{1-x}{x\left(1-x^{m}\right)}\left(1-\exp \left(\frac{x\left(1-x^{m}\right)}{x-1}\right)\right)\left(\frac{1-(m+1) x^{m}+m x^{m+1}}{(1-x)^{2}}\right)^{r},
\end{aligned}
$$

by (4.5), which gives (4.7).

\section{Polynomial generalization}

In this section, we briefly discuss a polynomial generalization of the sequence $\ell_{m}^{(r)}(n, k)$ based on a pair of statistics on $\mathcal{L}_{m}^{(r)}(n, k)$. Given a block $\mathcal{B}$ of $\lambda \in$ $\mathcal{L}_{m}^{(r)}(n, k)$, an element $i \in \mathcal{B}$ is said to be a left-to-right minimum if there exists no $j$ to the left of $i$ in $\mathcal{B}$ with $j<i$. If $\mathcal{B}$ is a special block of $\lambda$ containing say $b \in[r]$, then we will say that $i$ is a special block record low if (i) $i$ occurs to the left of $b$ in $\mathcal{B}$, with no element $j<i$ to the left of $i$, or (ii) $i$ occurs to the right of $b$ in $\mathcal{B}$, with no $j<i$ occurring between $b$ and $i$. Let $\operatorname{rec}^{\prime}(\lambda)$ denote the total number of special block record lows in all of its special blocks. Let $n \min (\lambda)$ denote the number of elements of $[r+1, r+n]$ either (a) belonging to a non-special block and not a left-to-right minimum, or (b) belonging to a special block and not a special block record low. For example, if $n=12, k=2, r=3, m=5$ and

$$
\lambda=\{10,7,1,12\},\{2\},\{5,15,3,6,8\},\{4,14,11\},\{13,9\} \in \mathcal{L}_{5}^{(3)}(12,2),
$$

then $\operatorname{rec}^{\prime}(\lambda)=5$ (the enumerated elements being 10, 7, 12, 5 and 6) and $\operatorname{nmin}(\lambda)=$ 4 (the elements being 15, 8, 14 and 11). Note that minimal elements in all blocks and left-to-right minima in non-special blocks are among those excluded from the counts of both statistics, whence $0 \leq n \min (\lambda)+\operatorname{rec}^{\prime}(\lambda) \leq n-k$ with all values in this range being realized. Define the joint distribution polynomial for the $\mathrm{nmin}$ and $r e c^{\prime}$ statistics on $\mathcal{L}_{m}^{(r)}(n, k)$ by

$$
\ell_{m}^{(r)}(n, k ; a, b)=\sum_{\lambda \in \mathcal{L}_{m}^{(r)}(n, k)} a^{n \min (\lambda)} b^{r e c^{\prime}(\lambda)} .
$$

See [13] for a related generalization of the Lah numbers.

Let $[a, b]_{j}=\prod_{j=1}^{m}(a j+b)$ if $j \geq 1$, with $[a, b]_{0}=1$. Considering whether or not the element $n+r$ forms its own block within a member of $\mathcal{L}_{m}^{(r)}(n, k)$, and if not, considering further cases based on whether $n+r$ follows directly a member of $[r+1, r+n-1]$ or starts a non-special block or is a special block record low yields the recurrence

$$
\ell_{m}^{(r)}(n, k ; a, b)=\ell_{m}^{(r)}(n-1, k-1 ; a, b)+(a(n-1)+k+2 b r) \ell_{m}^{(r)}(n-1, k ; a, b)
$$




$$
\begin{aligned}
& -[a, 1]_{m}\left(\begin{array}{c}
n-1 \\
m
\end{array}\right) \ell_{m}^{(r)}(n-m-1, k-1 ; a, b) \\
& -2 b r[a, 2 b]_{m-1}\left(\begin{array}{c}
n-1 \\
m-1
\end{array}\right) \ell_{m}^{(r-1)}(n-m, k ; a, b),
\end{aligned}
$$

which reduces to (3.1) when $a=b=1$. Note that it is possible to consider a further polynomial generalization wherein the $k \ell_{m}^{(r)}(n-1, k ; a, b)$ term in (5.1) is multiplied by an indeterminate $c$. However, the statistic marked by $c$ in this case can be obtained as $n-k-n \min (\lambda)-\operatorname{rec}^{\prime}(\lambda)$ for all $\lambda$. Thus, we may assume without loss of generality that one of $a, b$ or $c$ equals 1 , and it is most convenient here to take $c=1$.

Several of the properties shown in prior sections can be extended to the polynomial case. For example, generalizing the arguments used to show (3.2) and (3.8) respectively yields

$$
\begin{aligned}
\ell_{m}^{(r)}(n, k ; a, b)=n ! \sum_{i=0}^{k} \sum_{j=0}^{r} & \frac{(2 b)^{j}[a, 1]_{m-1}^{i}[a, 2 b]_{m-2}^{j}}{i !(m !)^{i}[(m-1) !]^{j}(n-m i-(m-1) j) !}\left(\begin{array}{l}
r \\
j
\end{array}\right) \\
& \times \ell_{m-1}^{(r-j)}(n-m i-(m-1) j, k-i ; a, b)
\end{aligned}
$$

and

$$
\begin{aligned}
\sum_{j=1}^{n}(j+p & +2 b r)[a, j+p+2 b r]_{k-1} \ell_{m}^{(r)}(n, j ; a, b) \\
& =\sum_{i=0}^{n} \sum_{s=0}^{p}(i+s) !\left(\begin{array}{c}
p \\
s
\end{array}\right) \ell^{(r)}(k, i+s ; a, b) L_{m}^{(r)}(n, i ; a, b),
\end{aligned}
$$

where $L_{m}^{(r)}(n, k ; a, b)=\sum_{j=k}^{n}\left(\begin{array}{l}j \\ k\end{array}\right) \ell_{m}^{(r)}(n, j ; a, b)$. One can also generalize (3.6) if the second sum in (3.6) is expressed instead using multiple indices which yields

$$
\begin{aligned}
\ell_{m}^{(r)}(n, k ; a, b)= & \sum_{j=1}^{r}(-1)^{j-1}\left(\begin{array}{l}
r \\
j
\end{array}\right) \ell_{m}^{(r-j)}(n, k ; a, b) \\
& +\sum_{\substack{i_{1}+\ldots+i_{r} \leq n-k \\
1 \leq i_{j} \leq m-1}}\left(\begin{array}{c}
n \\
i_{1}, \ldots, i_{r}, n-\sum_{j=1}^{r} i_{j}
\end{array}\right)\left((2 b)^{r} \prod_{j=1}^{r}[a, 2 b]_{i_{j}-1}\right) \\
& \times \ell_{m}^{(0)}\left(n-\sum_{j=1}^{r} i_{j}, k ; a, b\right) .
\end{aligned}
$$

Note that it is possible to write a recurrence for the multi-sum occurring in (5.4) that is analogous to (5.1) above (here, one would need an extra term $2 b r \ell_{m}^{(r-1)}(n-$ $1, k ; a, b)$ and assume $m \geq 2$ ).

We conclude by suggesting some further problems to consider. First, it would be interesting to find polynomial generalizations of formulas (3.4) and (4.1). The 
unimodality of $\ell_{m}^{(r)}(n, k)$ could be considered as well as for what $k$ the maximum value is achieved when $n$ is fixed. While the $r$-Lah numbers are log-concave (see, e.g., [16]), it seems to be more difficult to establish this fact for $\ell_{m}^{(r)}(n, k)$ or, more generally, for $\ell_{m}^{(r)}(n, k ; a, b)$ when $a$ and $b$ are positive real numbers, due to the more complicated formulas that are involved. Finally, it would be interesting to find an orthogonality relation for $\ell_{m}^{(r)}(n, k)$ that has as its limiting case when $m \rightarrow \infty$ the known orthogonality formula for the $r$-Lah numbers (see [16, Corollary 3.1]).

\section{References}

[1] Belbachir, H., Belkhir, A., Cross recurrence relations for $r$-Lah numbers, Ars. Combin. 110 (2013), 199-203.

[2] Belbachir, H., BousbaA, I.E., Associated Lah numbers and $r$-Stirling numbers, arXiv:1404.5573v2, 2014.

[3] Belbachir, H., BousbaA, I.E., Combinatorial identities for the $r$-Lah numbers, Ars Combin. 115 (2014), 453-458.

[4] Broder, A.Z., The $r$-Stirling numbers, Discrete Math. 49 (1984), 241-259.

https://doi.org/10.1016/0012-365x (84)90161-4

[5] Cheon, G.-S., Jung, J.-H., r-Whitney numbers of Dowling lattices, Discrete Math. 312 (2012), 2337-2348.

https://doi.org/10.1016/j.disc.2012.04.001

[6] Chor, J.Y., Multi-restrained Stirling numbers, Ars Combin. 120 (2015), 113-127.

[7] Graham, R.L., Knuth, D.E., Patashnik, O., Concrete Mathematics: A Foundation for Computer Science, second edition, Addison-Wesley, Boston, 1994. https://doi.org/10.2307/3619021

[8] KIm, D.S., KIm, T., Kwon, H.-I., Fourier series of $r$-derangement and higher-order derangement functions, Adv. Stud. Contemp. Math. 28(1) (2018), 1-11.

[9] Komatsu, T., Incomplete poly-Cauchy numbers, Monatsh. Math. 180 (2016), 271288.

https://doi.org/10.1007/s00605-015-0810-z

[10] Komatsu, T., Liptai, K., Mezö, I., Incomplete poly-Bernoulli numbers associated with incomplete Stirling numbers, arXiv:1510.05799v2, 2015.

[11] Komatsu, T., Mezö, I., Szalay, L., Incomplete Cauchy numbers, Acta Math. Hungar. 149(2) (2016), 306-323. https://doi.org/10.1007/s10474-016-0616-z

[12] LAH, I., A new kind of numbers and its application in the actuarial mathematics, Bol. Inst. Actuár. Port. 9 (1954), 7-15.

[13] Mansour, T., Shattuck, M., A generalized class of restricted Stirling and Lah numbers, Math. Slovaca 68:4 (2018), 727-740.

https://doi.org/10.1515/ms-2017-0140

[14] Merris, R., The p-Stirling numbers, Turkish J. Math. 24 (2000), 379-399. 
[15] Minoubi, M., Rahmani, M., The partial $r$-Bell polynomials, Afrika Mat. 28(7-8) (2017), 1167-1183.

https://doi.org/10.1007/s13370-017-0510-z

[16] Nyul, G., RÁcz, G., The r-Lah numbers, Discrete Math. 338 (2015), 1660-1666. https://doi.org/10.1016/j.disc.2014.03.029

[17] Shattuck, M., Generalized r-Lah numbers, Proc. Indian Acad. Sci. (Math. Sci.) 126(4) (2016), 461-478.

https://doi.org/10.1007/s12044-016-0309-0

[18] Shattuck, M., Wagner, C., Parity theorems for statistics on lattice paths and Laguerre configurations, J. Integer Seq. 8 (2005), Art. 5.5.1.

[19] Slonne, N.J., On-line Encyclopedia of Integer Sequences, published electronically at http://oeis.org (2010).

[20] Wang, C.-Y., Miska, P., Mezö, I., The $r$-derangement numbers, Discrete Math. 340(7) (2017), 1681-1692.

https://doi.org/10.1016/j.disc.2016.10.012 